Acta Crystallographica Section E

\section{Structure Reports}

Online

ISSN 1600-5368

\section{4-(Piperidin-1-yl)-4H-benzo[b]tetrazolo- $[1,5-d][1,4]$ diazepin-5(6H)-one}

\section{Gary S. Nichol, ${ }^{a *}$ Zhigang Xu, $^{\mathrm{b}}$ Christine E. Kaiser ${ }^{\mathrm{b}}$ and Christopher Hulme ${ }^{\text {b }}$}

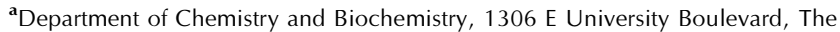
University of Arizona, Tucson, AZ 85721, USA, and ${ }^{\mathbf{b}}$ Southwest Center for Drug, Discovery and Development, College of Pharmacy, BIO5 Institute, The University of Arizona, Tucson, AZ 85721, USA

Correspondence e-mail: gsnichol@email.arizona.edu

Received 15 November 2010; accepted 29 November 2010

Key indicators: single-crystal X-ray study; $T=100 \mathrm{k}$; mean $\sigma(\mathrm{c}-\mathrm{C})=0.001 \mathrm{a} ; R$ factor $=$ $0.043 ; w R$ factor $=0.123 ;$ data-to-parameter ratio $=24.0$.

There are two crystallographically unique molecules present in the asymmetric unit of the title compound, $\mathrm{C}_{14} \mathrm{H}_{16} \mathrm{~N}_{6} \mathrm{O}$; in both molecules, the seven-membered diazepinone ring adopts a boat-like conformation and the chair conformation piperidine ring is an axial substituent on the diazepinone ring. In the crystal, each molecule forms hydrogen bonds with its respective symmetry equivalents. Hydrogen bonding between molecule $A$ and symmetry equivalents forms two ring motifs, the first formed by inversion-related $\mathrm{n}-\mathrm{h} \cdots \mathrm{o}$ interactions and the second formed by $\mathrm{c}-\mathrm{h} \cdots \mathrm{o}$ and $\mathrm{c}-\mathrm{h} \cdots \mathrm{n}$ interactions. the combination of both ring motifs results in the formation of an infinite double tape, which propagates in the $a$-axis direction. hydrogen bonding between molecule $B$ and symmetry equivalents forms one ring motif by inversion-related $n-$ $\mathrm{h} \cdots \mathrm{o}$ interactions and a second ring motif by $\mathrm{c}-\mathrm{h} \cdots \mathrm{o}$ interactions, which propagate as a single tape parallel with the $c$ axis.

\section{Related literature}

The structure of the title compound was determined as part of a larger study on development of synthetic methods for highthroughput medicinal chemistry. For background to the use of multi-component reactions in high-throughput medicinal chemistry, see: Gunawan et al. (2010); hulme \& dietrich (2009); hulme \& gore (2003). for the ugi reaction, see: ugi \& steinbrückner (1961). for graph-set notation for hydrogen bonding, see: bernstein et al. (1995) and puckering parameters, see: cremer \& pople (1975).

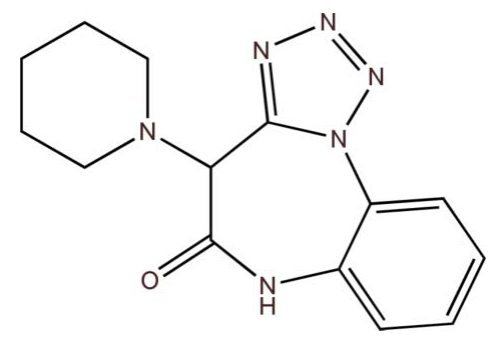

\section{Experimental}

\section{Crystal data \\ $\mathrm{C}_{14} \mathrm{H}_{16} \mathrm{~N}_{6} \mathrm{O}$ \\ $M_{r}=284.33$ \\ Triclinic, $P \overline{1}$ \\ $a=8.8210(7)$ å \\ $b=13.1802(10)$ å \\ $c=13.4476(11)$ å \\ $\alpha=105.549$ (2) \\ $\beta=99.490(2)^{\circ}$}

\section{Data collection} diffractometer

Absorption correction: numerical (SADABS; sheldrick, 1996)

$T_{\min }=0.965, T_{\max }=0.992$

Refinement

$R\left[F^{2}>2 \sigma\left(F^{2}\right)\right]=0.043$

$w R\left(F^{2}\right)=0.123$

$S=1.05$

12177 reflections
Bruker Kappa APEXII DUO CCD

$$
\begin{aligned}
& \gamma=106.623(2)^{\circ} \\
& V=1392.99(19) \mathrm{a}^{3} \\
& Z=4 \\
& \text { Mo } K \alpha \text { radiation } \\
& \mu=0.09 \mathrm{~mm}^{-1} \\
& T=100 \mathrm{k} \\
& 0.39 \times 0.28 \times 0.09 \mathrm{~mm}
\end{aligned}
$$

51078 measured reflections 12177 independent reflections 9733 reflections with $I>2 \sigma(I)$ $R_{\text {int }}=0.029$

507 parameters

All $\mathrm{H}$-atom parameters refined

$\Delta \rho_{\max }=0.59 \mathrm{e} \AA^{-3}$

$\Delta \rho_{\min }=-0.23 \mathrm{e}^{-3}$
Table 1

Hydrogen-bond geometry $\left(\AA{ }^{\circ}\right)$.

\begin{tabular}{lllll}
\hline$D-\mathrm{h} \cdots A$ & $D-\mathrm{h}$ & $\mathrm{H} \cdots A$ & $D \cdots A$ & $D-\mathrm{h} \cdots A$ \\
\hline $\mathrm{N} 1-\mathrm{H} 1 N \cdots \mathrm{o} 1^{\mathrm{i}}$ & $0.850(14)$ & $2.069(14)$ & $2.9089(9)$ & $169.4(13)$ \\
$\mathrm{N} 51-\mathrm{H} 51 N \cdots \mathrm{o} 1^{\mathrm{ii}}$ & $0.886(16)$ & $1.929(16)$ & $2.8116(10)$ & $173.6(14)$ \\
$\mathrm{C} 6-\mathrm{H} 6 \cdots \mathrm{N} 2^{\mathrm{iii}}$ & $0.936(15)$ & $2.531(15)$ & $3.4638(11)$ & $174.8(12)$ \\
$\mathrm{C} 7-\mathrm{H} 7 \cdots \mathrm{O} 1^{\text {iii }}$ & $0.947(15)$ & $2.406(15)$ & $3.3394(10)$ & $168.4(13)$ \\
$\mathrm{C} 55-\mathrm{H} 55 \cdots \mathrm{N} 54^{\text {iv }}$ & $0.974(13)$ & $2.548(13)$ & $3.2293(11)$ & $127.0(10)$ \\
\hline
\end{tabular}

Symmetry codes: (i) $-x+1,-y,-z+1$; (ii) $-x+1,-y+1,-z$; (iii) $x+1, y, z$; (iv) $-x+1,-y+1,-z+1$.

Data collection: APEX2 (bruker, 2007); cell refinement: SAINT (bruker, 2007); data reduction: $S A I N T$; $\operatorname{program}(\mathrm{s})$ used to solve structure: SHELXTL (sheldrick, 2008); program(s) used to refine structure: SHELXTL; molecular graphics: ORTEP-3 for Windows (farrugia, 1997) and Mercury (macrae et al. 2008); software used to prepare material for publication: SHELXTL and local programs.

The diffractometer was purchased with funding from NSF grant CHE-0741837.

Supplementary data and figures for this paper are available from the IUCr electronic archives (Reference: KJ2167). 


\section{organic compounds}

\section{References}

Bernstein, J., Davis, R. E., Shimoni, L. \& Chang, N.-L. (1995). Angew. Chem. Int. Ed. Engl. 34, 1555-1573.

Bruker (2007). APEX2 and SAINT. bruker axs inc., madison, wisconsin, usa. Cremer, D. \& Pople, J. A. (1975). J. Am. Chem. Soc. 97, 1354-1358.

Farrugia, L. J. (1997). J. Appl. Cryst. 30, 565.

Gunawan, S., Nichol, G. S., Chappeta, S., Dietrich, J. \& Hulme, C. (2010). Tetrahedron Lett. 51, 4689-4692
Hulme, C. \& Dietrich, J. (2009). Mol. Divers. 13, 195-207.

Hulme, C. \& Gore, V. (2003). Curr. Med. Chem. 10, 51-80.

Macrae, C. F., Bruno, I. J., Chisholm, J. A., Edgington, P. R., McCabe, P., Pidcock, E., Rodriguez-Monge, L., Taylor, R., van de Streek, J. \& Wood, P. A. (2008). J. Appl. Cryst. 41, 466-470.

Sheldrick, G. M. (1996). SADABS. university of göttingen, germany.

Sheldrick, G. M. (2008). Acta Cryst. a64, 112-122.

Ugi, I. \& Steinbrückner, C. (1961). Chem. Ber. 94, 734-742. 


\section{supporting information}

Acta Cryst. (2011). E67, o23-o24 [https://doi.org/10.1107/S1600536810049950]

\section{4-(Piperidin-1-yl)-4H-benzo[b] tetrazolo[1,5-d][1,4]diazepin-5(6H)-one}

\section{Gary S. Nichol, Zhigang Xu, Christine E. Kaiser and Christopher Hulme}

\section{S1. Comment}

At present there is a huge need for unique small molecules in the lead development stages of drug discovery. In this process, speed is paramount, and the development of high speed parallel synthesis in concert with isocyanide based multi-component reactions (MCRs) has enabled a revolution in high-throughput medicinal chemistry (Gunawan et al., (2010); Hulme \& Dietrich (2009); Hulme \& Gore (2003)). Following this theme, a novel two step solution phase protocol for the synthesis of an array of tricyclic fused tetrazole-benzodiazepines was recently investigated (Figure 1). The methodology employs ortho- $N$-Boc benzylisonitriles 1 and ethyl glyoxylate $\mathbf{2}$ in the 4-component TMS-N $\mathrm{N}_{3}$ modified Ugi reaction (Ugi \& Steinbrückner, 1961) to assemble the desired product 3. Subsequent treatment with trifluoroacetic acid unmasks an internal amino nucleophile and promotes cyclization to form the diazepine ring of the generic structure 4. Here we report the crystal structure of 4.

The asymmetric unit of $\mathbf{4}$ is shown in Figure 2. There are two crystallographically unique molecules in the asymmetric unit; the molecule composed of atoms $\mathrm{O} 1$ to $\mathrm{C} 14$ will henceforth be referred to as "molecule $\mathrm{A}$ " and the molecule composed of atoms $\mathrm{O} 51$ to $\mathrm{C} 64$ referred to as "molecule B". Where appropriate, discussion will be limited to molecule A with results for molecule B presented in square brackets. Molecular dimensions are unexceptional.

The molecule adopts a U-shaped conformation in which the 7-membered diazepinone ring has adopted a boat-like conformation (total Q parameter 0.8021 (8) $\AA$ [0.8177 (9) $\AA]$; Cremer \& Pople (1975)) and the chair conformation piperidinyl ring is an axial substituent on the diazepinone ring. Both molecules have a very similar overall shape as shown by an overlay, fitting N1, N5, C4 > C9 with N51, N55, C54 > C59 (these representing the largest planar moiety in the structure, Figure 3).

In the crystal each molecule forms hydrogen bonds with its respective symmetry equivalents. Hydrogen bonding between molecule A and symmetry equivalents forms two ring motifs (Bernstein et al., 1995), an $R^{2}{ }_{2}(8)$ motif formed by inversion-related $\mathrm{N}-\mathrm{H} \cdots \mathrm{O}$ interactions and an $R_{2}{ }_{2}(9)$ motif formed by $\mathrm{C}-\mathrm{H} \cdots \mathrm{O}$ and $\mathrm{C}-\mathrm{H} \cdots \mathrm{N}$ interactions. The combination of both ring motifs results in the formation of an infinite double tape which propagates in the $a$ axis direction (Figure 4). Hydrogen bonding between molecule B and symmetry equivalents forms one ring motif composed of an $R^{2}{ }_{2}(8)$ motif formed by inversion-related $\mathrm{N}-\mathrm{H} \cdots \mathrm{O}$ interactions and an $R^{2}{ }_{2}(10)$ motif formed by $\mathrm{C}-\mathrm{H} \cdots \mathrm{O}$ interactions (Figure 5). This propagates as a single tape parallel with the $c$ axis.

\section{S2. Experimental}

A solution of piperidine $(0.017 \mathrm{~g}, 0.20 \mathrm{mmol})$ and ethyl glyoxylate $(0.04 \mathrm{ml}, 50 \%$ in toluene, $0.20 \mathrm{mmol})$ in methanol $(0.5 \mathrm{ml})$ were stirred at room temperature. After 5 minutes, ortho- $N$-Boc-phenylisonitrile $(0.0436 \mathrm{~g}, 0.20 \mathrm{mmol})$ and trimethylsilylazide $(0.023 \mathrm{~g}, 0.20 \mathrm{mmol})$ was added dropwise to the above solution and stirred at room temperature for 23 $\mathrm{h}$. The solvent was evaporated in vacuo and the product was purified using column chromatography (5-30\% Hexane/Ethyl Acetate) to afford the desired Ugi product ( $0.056 \mathrm{~g}, 0.20 \mathrm{mmol}, 65 \%)$ as colorless oil. The purified Ugi 
product was treated with $10 \%$ trifluoroacetic acid in dichloroethane $(4 \mathrm{ml})$ and irradiated in a Biotage Initiator\&trade; for 10 minutes at $120^{\circ} \mathrm{C}$. The organic layer was washed with $1 M \mathrm{NaHCO}_{3}(3 \times 5 \mathrm{ml})$ and dried $\left(\mathrm{MgSO}_{4}\right)$. The solvent was evaporated in vacuo and purified by column chromatography (0-50\% Hexane/Ethyl Acetate) to afford the desired product $(0.030 \mathrm{~g}, 0.116 \mathrm{mmol}, 92 \%)$ as a white solid.

\section{S3. Refinement}

All hydrogen atoms were located in a difference Fourier map and are freely refined.

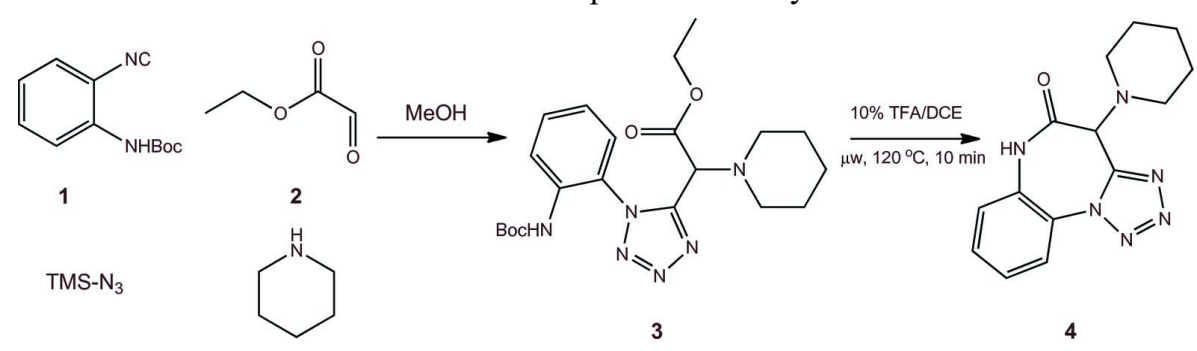

Figure 1

The synthetic route to 4 .
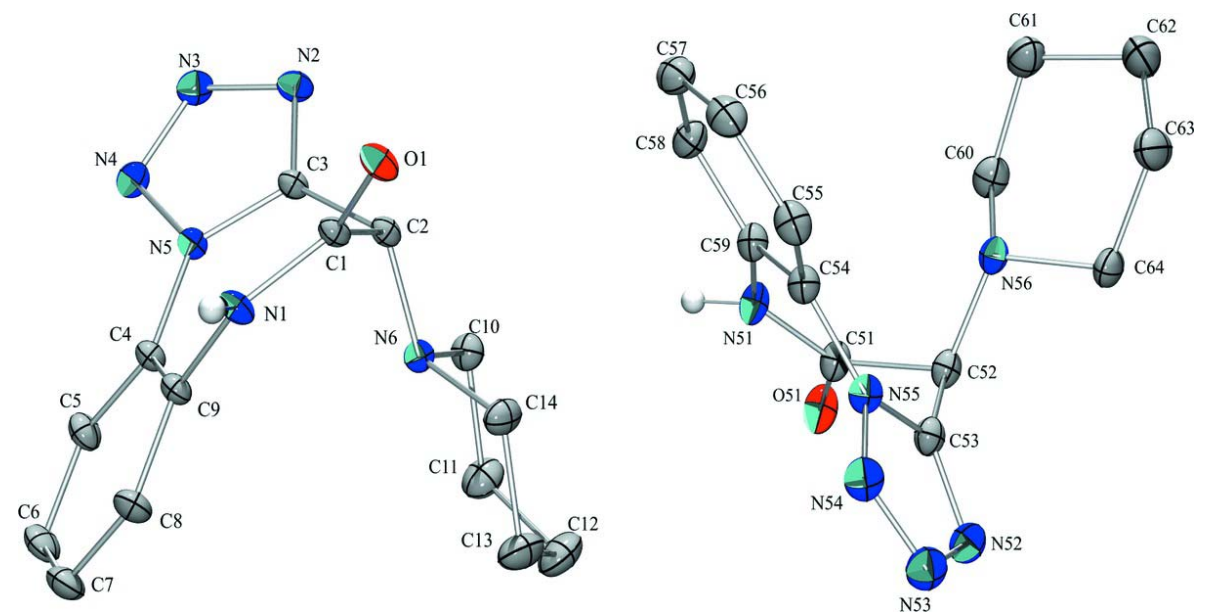

Figure 2

The asymmetric unit of $\mathbf{4}$ with displacement ellipsoids at the $50 \%$ probability level and C-bound $\mathrm{H}$ atoms omitted. 


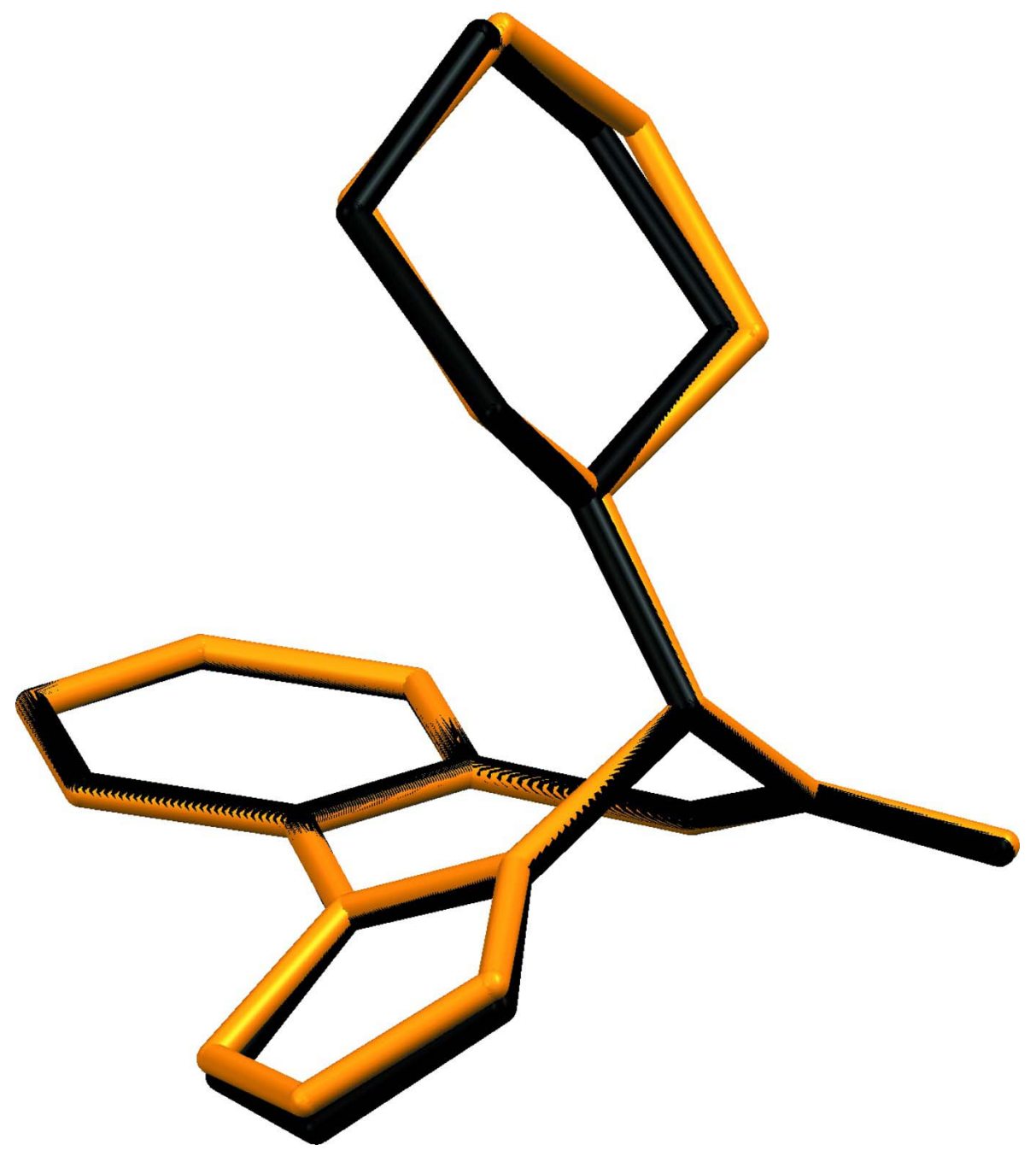

Figure 3

An overlay of molecule A (orange) and molecule B (black), r.m.s. deviation $=0.0185 \AA$ ), in 4.

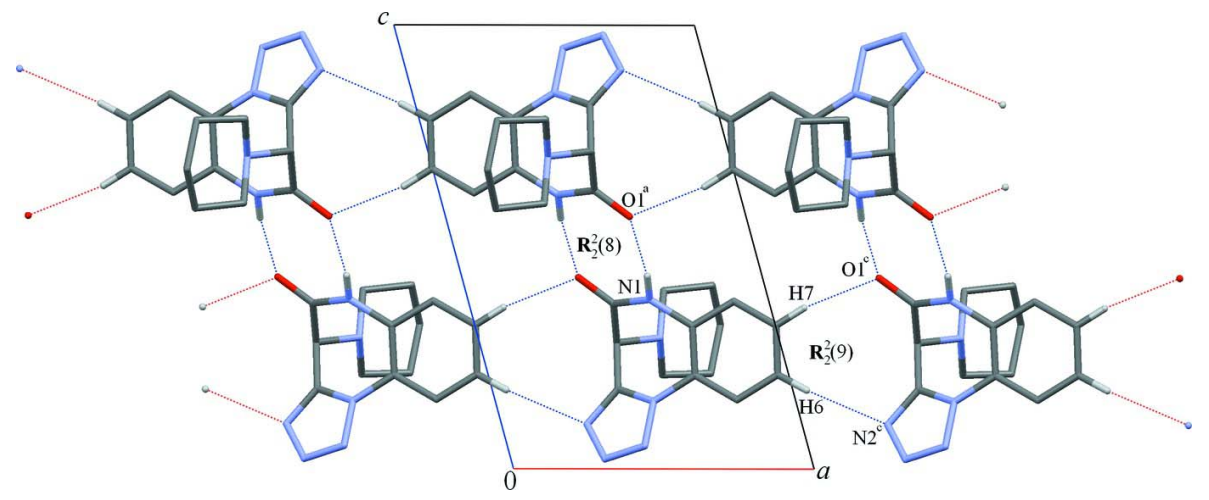

Figure 4

Hydrogen bonding patterns (dotted blue lines) formed by molecule A in 4 . Symmetry operations: a, $-x+1,-y,-z+1 ; \mathrm{c}, x$ $+1, y, z$. 


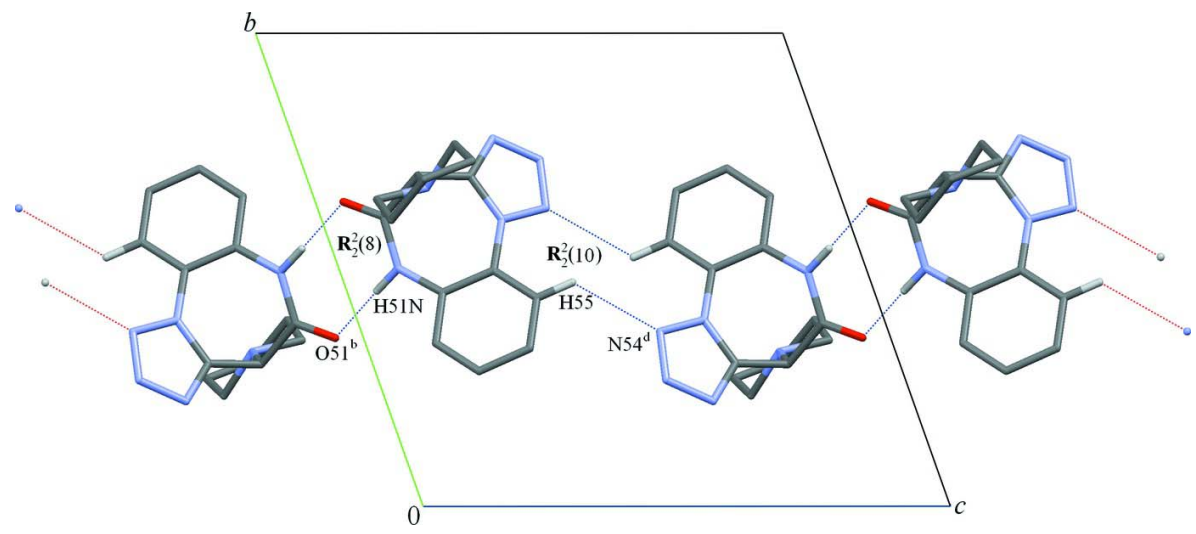

Figure 5

Hydrogen bonding patterns (dotted blue lines) formed by molecule B in 4 . Symmetry operations: $\mathrm{b},-x+1,-y+1,-z ; \mathrm{d},-x$ $+1,-y+1,-z+1$.

\section{4-(Piperidin-1-yl)-4H-benzo[b]tetrazolo[1,5- $d][1,4]$ diazepin-5(6H)-one}

\section{Crystal data}

$\mathrm{C}_{14} \mathrm{H}_{16} \mathrm{~N}_{6} \mathrm{O}$

$M_{r}=284.33$

Triclinic, $P \overline{1}$

Hall symbol: -P 1

$a=8.8210(7) \AA$

$b=13.1802(10) \AA$

$c=13.4476(11) \AA$

$\alpha=105.549(2)^{\circ}$

$\beta=99.490(2)^{\circ}$

$\gamma=106.623(2)^{\circ}$

$V=1392.99(19) \AA^{3}$

\section{Data collection}

Bruker Kappa APEXII DUO CCD diffractometer

Radiation source: fine-focus sealed tube with Miracol optics

Graphite monochromator

$\varphi$ and $\omega$ scans

Absorption correction: numerical

(SADABS; Sheldrick, 1996)

$T_{\min }=0.965, T_{\max }=0.992$

\section{Refinement}

Refinement on $F^{2}$

Least-squares matrix: full

$R\left[F^{2}>2 \sigma\left(F^{2}\right)\right]=0.043$

$w R\left(F^{2}\right)=0.123$

$S=1.05$

12177 reflections

507 parameters

0 restraints

Primary atom site location: structure-invariant direct methods
$Z=4$

$F(000)=600$

$D_{\mathrm{x}}=1.356 \mathrm{Mg} \mathrm{m}^{-3}$

Mo $K \alpha$ radiation, $\lambda=0.71073 \AA$

Cell parameters from 9970 reflections

$\theta=2.5-35.5^{\circ}$

$\mu=0.09 \mathrm{~mm}^{-1}$

$T=100 \mathrm{~K}$

Prism, colourless

$0.39 \times 0.28 \times 0.09 \mathrm{~mm}$

51078 measured reflections

12177 independent reflections

9733 reflections with $I>2 \sigma(I)$

$R_{\text {int }}=0.029$

$\theta_{\max }=35.0^{\circ}, \theta_{\min }=1.6^{\circ}$

$h=-8 \rightarrow 14$

$k=-21 \rightarrow 21$

$l=-21 \rightarrow 19$

Secondary atom site location: difference Fourier map

Hydrogen site location: difference Fourier map

All $\mathrm{H}$-atom parameters refined

$w=1 /\left[\sigma^{2}\left(F_{\mathrm{o}}^{2}\right)+(0.0711 P)^{2}+0.1991 P\right]$

where $P=\left(F_{\mathrm{o}}^{2}+2 F_{\mathrm{c}}^{2}\right) / 3$

$(\Delta / \sigma)_{\max }<0.001$

$\Delta \rho_{\max }=0.59 \mathrm{e}^{-3}$

$\Delta \rho_{\min }=-0.23$ e $\AA^{-3}$ 


\section{Special details}

Geometry. All e.s.d.'s (except the e.s.d. in the dihedral angle between two 1.s. planes) are estimated using the full covariance matrix. The cell e.s.d.'s are taken into account individually in the estimation of e.s.d.'s in distances, angles and torsion angles; correlations between e.s.d.'s in cell parameters are only used when they are defined by crystal symmetry. An approximate (isotropic) treatment of cell e.s.d.'s is used for estimating e.s.d.'s involving 1.s. planes.

Refinement. Refinement of $F^{2}$ against ALL reflections. The weighted $R$-factor $w R$ and goodness of fit $S$ are based on $F^{2}$, conventional $R$-factors $R$ are based on $F$, with $F$ set to zero for negative $F^{2}$. The threshold expression of $F^{2}>\sigma\left(F^{2}\right)$ is used only for calculating $R$-factors (gt) etc. and is not relevant to the choice of reflections for refinement. $R$-factors based on $F^{2}$ are statistically about twice as large as those based on $F$, and $R$ - factors based on ALL data will be even larger.

Fractional atomic coordinates and isotropic or equivalent isotropic displacement parameters $\left(\AA^{2}\right)$

\begin{tabular}{|c|c|c|c|c|}
\hline & $x$ & $y$ & $z$ & $U_{\text {iso }} * / U_{\text {eq }}$ \\
\hline $\mathrm{O} 1$ & $0.38761(7)$ & $0.05350(5)$ & $0.43124(5)$ & $0.01571(11)$ \\
\hline N1 & $0.60561(8)$ & $0.01832(6)$ & $0.38204(5)$ & $0.01320(11)$ \\
\hline $\mathrm{H} 1 \mathrm{~N}$ & $0.6179(16)$ & $-0.0054(11)$ & $0.4346(11)$ & $0.022(3)^{*}$ \\
\hline N2 & $0.28444(8)$ & $-0.02275(6)$ & $0.10021(5)$ & $0.01491(11)$ \\
\hline N3 & $0.31273(9)$ & $-0.10081(6)$ & $0.02234(5)$ & $0.01668(12)$ \\
\hline N4 & $0.45718(9)$ & $-0.10612(6)$ & $0.05193(5)$ & $0.01538(12)$ \\
\hline N5 & $0.52683(8)$ & $-0.02989(5)$ & $0.15250(5)$ & $0.01189(10)$ \\
\hline N6 & $0.60485(8)$ & $0.20364(5)$ & $0.29571(5)$ & $0.01251(11)$ \\
\hline $\mathrm{C} 1$ & $0.48112(9)$ & $0.05928(6)$ & $0.37255(6)$ & $0.01170(11)$ \\
\hline $\mathrm{C} 2$ & $0.45700(9)$ & $0.11124(6)$ & $0.28435(6)$ & $0.01159(11)$ \\
\hline $\mathrm{H} 2$ & $0.3595(15)$ & $0.1330(10)$ & $0.2855(10)$ & $0.017(3)^{*}$ \\
\hline $\mathrm{C} 3$ & $0.41921(9)$ & $0.02050(6)$ & $0.17984(6)$ & $0.01156(11)$ \\
\hline $\mathrm{C} 4$ & $0.68763(9)$ & $-0.01103(6)$ & $0.21140(6)$ & $0.01208(12)$ \\
\hline $\mathrm{C} 5$ & $0.80602(10)$ & $-0.02132(7)$ & $0.15637(7)$ & $0.01617(13)$ \\
\hline H5 & $0.7760(17)$ & $-0.0426(12)$ & $0.0765(11)$ & $0.027(3)^{*}$ \\
\hline C6 & $0.96362(10)$ & $-0.00308(7)$ & $0.21295(7)$ & $0.01873(14)$ \\
\hline H6 & 1.0457 (18) & $-0.0093(12)$ & $0.1781(12)$ & $0.030(3)^{*}$ \\
\hline $\mathrm{C} 7$ & $1.00262(10)$ & $0.02641(7)$ & $0.32418(7)$ & $0.01900(14)$ \\
\hline $\mathrm{H} 7$ & 1.1107 (19) & $0.0416(12)$ & $0.3635(12)$ & $0.033(4)^{*}$ \\
\hline $\mathrm{C} 8$ & $0.88362(9)$ & $0.03591(7)$ & $0.37831(7)$ & $0.01614(13)$ \\
\hline H8 & $0.9081(16)$ & $0.0536(11)$ & $0.4581(11)$ & $0.022(3)^{*}$ \\
\hline C9 & $0.72383(9)$ & $0.01681(6)$ & $0.32280(6)$ & $0.01220(12)$ \\
\hline $\mathrm{C} 10$ & $0.57954(10)$ & $0.25511(7)$ & $0.21242(6)$ & $0.01617(13)$ \\
\hline $\mathrm{H} 10 \mathrm{~A}$ & $0.5286(16)$ & $0.1938(11)$ & $0.1401(11)$ & $0.023(3)^{*}$ \\
\hline H10B & $0.4961(17)$ & $0.2921(11)$ & $0.2234(11)$ & $0.025(3)^{*}$ \\
\hline $\mathrm{C} 11$ & $0.74158(12)$ & $0.33854(7)$ & $0.21560(8)$ & $0.02248(16)$ \\
\hline H11A & $0.8144(18)$ & $0.2980(13)$ & $0.1966(12)$ & $0.033(4)^{*}$ \\
\hline H11 & $0.722(2)$ & $0.3742(13)$ & $0.1604(13)$ & $0.040(4)^{*}$ \\
\hline $\mathrm{C} 12$ & $0.81799(14)$ & $0.42864(8)$ & $0.32592(9)$ & 0.02767 (19) \\
\hline $\mathrm{H} 12 \mathrm{~A}$ & $0.753(2)$ & $0.4792(14)$ & $0.3395(13)$ & $0.039(4)^{*}$ \\
\hline H12B & $0.933(2)$ & $0.4793(14)$ & $0.3300(13)$ & $0.039(4)^{*}$ \\
\hline $\mathrm{C} 13$ & $0.82547(12)$ & $0.37556(8)$ & $0.41398(8)$ & $0.02422(17)$ \\
\hline H13A & 0.9033 (18) & $0.3335(12)$ & $0.4088(12)$ & $0.031(4)^{*}$ \\
\hline H13B & 0.8589 (17) & $0.4330(12)$ & $0.4846(11)$ & $0.027(3)^{*}$ \\
\hline C14 & $0.65953(10)$ & $0.28969(7)$ & $0.40202(6)$ & $0.01774(14)$ \\
\hline
\end{tabular}




\begin{tabular}{|c|c|c|c|c|}
\hline $\mathrm{H} 14 \mathrm{~A}$ & $0.6707(17)$ & $0.2514(11)$ & $0.4561(11)$ & $0.026(3)^{*}$ \\
\hline H14B & $0.5794(18)$ & $0.3278(12)$ & $0.4109(11)$ & $0.029(3)^{*}$ \\
\hline O51 & $0.52486(10)$ & $0.64389(6)$ & $0.05346(5)$ & $0.02384(13)$ \\
\hline N51 & $0.40757(10)$ & $0.50343(6)$ & $0.11293(6)$ & $0.01766(13)$ \\
\hline $\mathrm{H} 51 \mathrm{~N}$ & $0.4212(19)$ & $0.4560(13)$ & $0.0574(13)$ & $0.036(4)^{*}$ \\
\hline N52 & $0.67271(10)$ & $0.77756(6)$ & $0.37934(6)$ & $0.02130(14)$ \\
\hline N53 & $0.72173(10)$ & $0.73295(7)$ & $0.45426(6)$ & $0.02402(15)$ \\
\hline N54 & $0.62468(10)$ & $0.63061(7)$ & $0.43334(6)$ & $0.02147(14)$ \\
\hline N55 & $0.50786(9)$ & $0.60616(6)$ & $0.34204(5)$ & $0.01671(12)$ \\
\hline N56 & $0.26638(9)$ & $0.67324(6)$ & $0.22010(5)$ & $0.01605(12)$ \\
\hline C51 & $0.45755(11)$ & $0.61293(7)$ & $0.11881(6)$ & $0.01729(14)$ \\
\hline C52 & $0.43580(10)$ & $0.69955(6)$ & $0.21216(6)$ & $0.01637(13)$ \\
\hline H52 & $0.4837(16)$ & $0.7759(11)$ & $0.2025(10)$ & $0.020(3)^{*}$ \\
\hline C53 & $0.53997(10)$ & $0.69786(7)$ & $0.31100(6)$ & $0.01685(13)$ \\
\hline C54 & $0.38196(10)$ & $0.49889(6)$ & $0.29242(6)$ & $0.01599(13)$ \\
\hline C55 & $0.31368(11)$ & $0.44075(7)$ & $0.35588(7)$ & $0.01975(15)$ \\
\hline H55 & $0.3508(16)$ & $0.4735(11)$ & $0.4336(11)$ & $0.022(3)^{*}$ \\
\hline C56 & $0.19621(11)$ & $0.33379(8)$ & $0.30809(8)$ & $0.02194(16)$ \\
\hline H56 & $0.1541(17)$ & $0.2909(12)$ & $0.3505(11)$ & $0.027(3)^{*}$ \\
\hline C57 & $0.14557(11)$ & $0.28606(7)$ & $0.19694(8)$ & $0.02214(16)$ \\
\hline H57 & $0.0629(17)$ & $0.2098(12)$ & $0.1595(12)$ & $0.029(3)^{*}$ \\
\hline C58 & 0.21359 (11) & $0.34480(7)$ & $0.13379(7)$ & $0.01951(15)$ \\
\hline H58 & $0.1781(17)$ & $0.3104(12)$ & $0.0555(11)$ & $0.027(3)^{*}$ \\
\hline C59 & $0.33285(10)$ & $0.45203(6)$ & $0.18068(6)$ & $0.01597(13)$ \\
\hline C60 & $0.14703(12)$ & $0.64876(7)$ & $0.11863(7)$ & $0.02103(15)$ \\
\hline H60 & $0.1632(16)$ & $0.7177(11)$ & $0.1003(11)$ & $0.023(3)^{*}$ \\
\hline H60B & $0.1633(17)$ & $0.5889(12)$ & $0.0625(11)$ & $0.028(3)^{*}$ \\
\hline C61 & $-0.02563(12)$ & $0.60567(8)$ & $0.13221(8)$ & $0.02450(17)$ \\
\hline H61A & $-0.1061(19)$ & $0.5883(13)$ & $0.0638(13)$ & $0.038(4)^{*}$ \\
\hline H61B & $-0.0431(17)$ & $0.5330(12)$ & $0.1488(11)$ & $0.027(3)^{*}$ \\
\hline C62 & $-0.05395(13)$ & $0.69164(9)$ & $0.22197(9)$ & $0.0310(2)$ \\
\hline H62A & $-0.059(2)$ & $0.7574(15)$ & $0.1989(14)$ & $0.049(5)^{*}$ \\
\hline H62B & -0.164 (2) & $0.6604(14)$ & $0.2299(13)$ & $0.039(4) *$ \\
\hline C63 & $0.08227(13)$ & $0.73000(9)$ & $0.32478(8)$ & $0.02806(19)$ \\
\hline H63A & $0.075(2)$ & $0.6703(14)$ & $0.3558(13)$ & $0.039(4)^{*}$ \\
\hline H63B & $0.0736(19)$ & $0.7914(13)$ & $0.3791(13)$ & $0.038(4)^{*}$ \\
\hline C64 & $0.25074(11)$ & $0.76573(7)$ & $0.30339(7)$ & $0.02088(15)$ \\
\hline H64A & $0.3370(17)$ & $0.7863(11)$ & $0.3681(11)$ & $0.025(3)^{*}$ \\
\hline H64B & $0.2700(17)$ & $0.8337(12)$ & $0.2788(11)$ & $0.030(3)^{*}$ \\
\hline
\end{tabular}

Atomic displacement parameters $\left(\AA^{2}\right)$

\begin{tabular}{lllllll}
\hline & $U^{11}$ & $U^{22}$ & $U^{33}$ & $U^{12}$ & $U^{13}$ & $U^{23}$ \\
\hline O1 & $0.0141(2)$ & $0.0241(3)$ & $0.0131(2)$ & $0.0092(2)$ & $0.00717(19)$ & $0.0080(2)$ \\
N1 & $0.0118(2)$ & $0.0199(3)$ & $0.0118(2)$ & $0.0077(2)$ & $0.0050(2)$ & $0.0080(2)$ \\
N2 & $0.0124(3)$ & $0.0189(3)$ & $0.0118(3)$ & $0.0044(2)$ & $0.0018(2)$ & $0.0046(2)$ \\
N3 & $0.0167(3)$ & $0.0181(3)$ & $0.0123(3)$ & $0.0043(2)$ & $0.0021(2)$ & $0.0034(2)$ \\
N4 & $0.0179(3)$ & $0.0152(3)$ & $0.0108(2)$ & $0.0049(2)$ & $0.0028(2)$ & $0.0023(2)$
\end{tabular}




\begin{tabular}{|c|c|c|c|c|c|c|}
\hline N5 & $0.0120(2)$ & $0.0138(2)$ & $0.0102(2)$ & $0.0050(2)$ & 0.00305 (19) & 0.00371 (19) \\
\hline N6 & $0.0132(3)$ & $0.0130(2)$ & $0.0109(2)$ & $0.0040(2)$ & $0.0037(2)$ & $0.00355(19)$ \\
\hline $\mathrm{C} 1$ & $0.0102(3)$ & 0.0149 (3) & $0.0102(3)$ & $0.0048(2)$ & $0.0032(2)$ & $0.0038(2)$ \\
\hline $\mathrm{C} 2$ & $0.0109(3)$ & $0.0146(3)$ & $0.0103(3)$ & $0.0054(2)$ & $0.0035(2)$ & $0.0042(2)$ \\
\hline $\mathrm{C} 3$ & $0.0108(3)$ & $0.0142(3)$ & $0.0107(3)$ & $0.0047(2)$ & $0.0035(2)$ & $0.0050(2)$ \\
\hline $\mathrm{C} 4$ & $0.0105(3)$ & $0.0147(3)$ & $0.0127(3)$ & $0.0056(2)$ & $0.0041(2)$ & $0.0053(2)$ \\
\hline $\mathrm{C} 5$ & 0.0159 & $0.0203(3)$ & $0.0176(3)$ & $0.0096(3)$ & 0.0090 & $0.0084(3)$ \\
\hline C6 & $0.0142(3)$ & $0.0241(4)$ & $0.0242(4)$ & $0.0101(3)$ & $0.0104(3)$ & $0.0109(3)$ \\
\hline $\mathrm{C} 7$ & $0.0113(3)$ & $0.0238(4)$ & $0.0247(4)$ & 0.0079 & 0.0053 & $0.0099(3)$ \\
\hline $\mathrm{C} 8$ & $0.0114(3)$ & $0.0208(3)$ & $0.0168(3)$ & $0.0065(2)$ & $0.0026(2)$ & 0.0070 \\
\hline C9 & 0.0103 & 0.0150 & $0.0134(3)$ & $0.0055(2)$ & $0.0044(2)$ & 0.0059 (2) \\
\hline $\mathrm{C} 10$ & 0.0185 & 0.0160 & $0.0159(3)$ & 0.0063 & 0.0051 & $0.0076(2)$ \\
\hline $\mathrm{C} 11$ & $0.0251(4)$ & $0.0194(3)$ & $0.0221(4)$ & 0.0027 & $0.0097(3)$ & 0.0090 \\
\hline $\mathrm{C} 12$ & $0.0297(5)$ & 0.0174 & 0.0284 & -0.0010 & 0.0088 & 0.0050 \\
\hline $\mathrm{C} 13$ & 0.0205 & $0.0219(4)$ & 0.0203 & -0.0014 & 0.0043 & 0.0015 \\
\hline $\mathrm{C} 14$ & 0.0177 & $0.0176(3)$ & $0.0132(3)$ & 0.0027 & 0.0049 & $0.0010(2)$ \\
\hline O51 & $0.0383(4)$ & $0.0196(3)$ & $0.0197(3)$ & $0.0109(3)$ & $0.0165(3)$ & $0.0097(2)$ \\
\hline N51 & $0.0272(3)$ & $0.0141(3)$ & $0.0140(3)$ & $0.0077(2)$ & 0.0090 & $0.0054(2)$ \\
\hline N52 & $0.0236(3)$ & $0.0198(3)$ & $0.0173(3)$ & $0.0063(3)$ & $0.0042(3)$ & $0.0032(2)$ \\
\hline N53 & $0.0253(4)$ & $0.0247(3)$ & $0.0186(3)$ & $0.0083(3)$ & 0.0019 & $0.0044(3)$ \\
\hline N54 & $0.0247(4)$ & $0.0246(3)$ & $0.0143(3)$ & $0.0102(3)$ & 0.0018 & $0.0056(2)$ \\
\hline N55 & $0.0202(3)$ & $0.0180(3)$ & $0.0126(3)$ & $0.0074(2)$ & $0.0042(2)$ & $0.0055(2)$ \\
\hline N56 & 0.0200 & $0.0147(3)$ & 0.0128 & $0.0066(2)$ & $0.0037(2)$ & $0.0033(2)$ \\
\hline C51 & $0.0245(4)$ & $0.0157(3)$ & 0.0137 (3) & 0.0078 & 0.0070 & $0.0059(2)$ \\
\hline C52 & 0.0221 & $0.0142(3)$ & 0.0135 & $0.0063(3)$ & 0.0059 & $0.0049(2)$ \\
\hline C53 & $0.0206(3)$ & $0.0162(3)$ & $0.0143(3)$ & 0.0070 & 0.0060 & $0.0044(2)$ \\
\hline C54 & $0.0192(3)$ & 0.0163 & $0.0150(3)$ & 0.0075 & 0.0059 & $0.0068(2)$ \\
\hline $\mathrm{C} 55$ & 0.0243 & $0.0234(4)$ & $0.0189(3)$ & 0.0120 & $0.0102(3)$ & 0.0119 (3) \\
\hline C56 & $0.0226(4)$ & $0.0242(4)$ & $0.0269(4)$ & 0.0099 (3) & 0.0119 & 0.0159 \\
\hline $\mathrm{C} 57$ & 0.0207 (4) & 0.0194 (3) & 0.0278 & 0.0057 (3) & $0.0071(3)$ & $0.0111(3)$ \\
\hline C58 & $0.0222(4)$ & $0.0165(3)$ & $0.0190(3)$ & 0.0057 & $0.0042(3)$ & 0.0065 \\
\hline C59 & 0.0202 & 0.0158 & 0.0150 & 0.0078 & 0.0065 & $0.0071(2)$ \\
\hline C60 & $0.0260(4)$ & 0.0214 & 0.0149 & 0.0088 & 0.0022 & 0.0060 \\
\hline C61 & 0.0238 & $0.0219(4)$ & 0.0229 & 0.0070 & 0.0005 & $0.0041(3)$ \\
\hline C62 & $0.0237(4)$ & $0.0301(5)$ & $0.0333(5)$ & $0.0114(4)$ & $0.0046(4)$ & $0.0010(4)$ \\
\hline C63 & $0.0242(4)$ & $0.0281(4)$ & 0.0259 (4) & 0.0074 & 0.0098 & $-0.0006(3)$ \\
\hline C64 & $0.0229(4)$ & $0.0161(3)$ & $0.0193(4)$ & 0.0058 & 0.0058 & -0.0001 \\
\hline
\end{tabular}

Geometric parameters $\left(A,{ }^{o}\right)$

\begin{tabular}{llll}
\hline $\mathrm{O} 1-\mathrm{C} 1$ & $1.2329(9)$ & $\mathrm{O} 51-\mathrm{C} 51$ & $1.2317(10)$ \\
$\mathrm{N} 1-\mathrm{H} 1 \mathrm{~N}$ & $0.850(14)$ & $\mathrm{N} 51-\mathrm{H} 51 \mathrm{~N}$ & $0.886(16)$ \\
$\mathrm{N} 1-\mathrm{C} 1$ & $1.3578(9)$ & $\mathrm{N} 51-\mathrm{C} 51$ & $1.3589(10)$ \\
$\mathrm{N} 1-\mathrm{C} 9$ & $1.4142(10)$ & $\mathrm{N} 51-\mathrm{C} 59$ & $1.4131(11)$ \\
$\mathrm{N} 2-\mathrm{N} 3$ & $1.3686(10)$ & $\mathrm{N} 52-\mathrm{N} 53$ & $1.3663(11)$ \\
$\mathrm{N} 2-\mathrm{C} 3$ & $1.3229(9)$ & $\mathrm{N} 52-\mathrm{C} 53$ & $1.3202(11)$ \\
$\mathrm{N} 3-\mathrm{N} 4$ & $1.2983(10)$ & $\mathrm{N} 53-\mathrm{N} 54$ & $1.2992(12)$ \\
$\mathrm{N} 4-\mathrm{N} 5$ & $1.3640(9)$ & $\mathrm{N} 54-\mathrm{N} 55$ & $1.3615(10)$
\end{tabular}




\begin{tabular}{|c|c|c|c|}
\hline $\mathrm{N} 5-\mathrm{C} 3$ & $1.3499(9)$ & $\mathrm{N} 55-\mathrm{C} 53$ & $1.3524(11)$ \\
\hline $\mathrm{N} 5-\mathrm{C} 4$ & $1.4225(9)$ & $\mathrm{N} 55-\mathrm{C} 54$ & $1.4244(11)$ \\
\hline $\mathrm{N} 6-\mathrm{C} 2$ & $1.4605(10)$ & $\mathrm{N} 56-\mathrm{C} 52$ & $1.4632(11)$ \\
\hline $\mathrm{N} 6-\mathrm{C} 10$ & $1.4752(10)$ & $\mathrm{N} 56-\mathrm{C} 60$ & $1.4731(11)$ \\
\hline $\mathrm{N} 6-\mathrm{C} 14$ & $1.4674(10)$ & N56-C64 & $1.4748(11)$ \\
\hline $\mathrm{C} 1-\mathrm{C} 2$ & $1.5351(10)$ & $\mathrm{C} 51-\mathrm{C} 52$ & $1.5338(11)$ \\
\hline $\mathrm{C} 2-\mathrm{H} 2$ & $0.982(12)$ & $\mathrm{C} 52-\mathrm{H} 52$ & $1.026(13)$ \\
\hline $\mathrm{C} 2-\mathrm{C} 3$ & $1.4944(10)$ & $\mathrm{C} 52-\mathrm{C} 53$ & $1.4955(11)$ \\
\hline $\mathrm{C} 4-\mathrm{C} 5$ & $1.3923(10)$ & $\mathrm{C} 54-\mathrm{C} 55$ & $1.3908(11)$ \\
\hline $\mathrm{C} 4-\mathrm{C} 9$ & $1.4004(10)$ & $\mathrm{C} 54-\mathrm{C} 59$ & $1.4005(11)$ \\
\hline $\mathrm{C} 5-\mathrm{H} 5$ & $1.002(14)$ & C55-H55 & $0.974(13)$ \\
\hline $\mathrm{C} 5-\mathrm{C} 6$ & $1.3897(11)$ & $\mathrm{C} 55-\mathrm{C} 56$ & $1.3904(13)$ \\
\hline $\mathrm{C} 6-\mathrm{H} 6$ & $0.936(15)$ & C56-H56 & $0.951(14)$ \\
\hline $\mathrm{C} 6-\mathrm{C} 7$ & $1.3959(13)$ & $\mathrm{C} 56-\mathrm{C} 57$ & $1.3943(14)$ \\
\hline $\mathrm{C} 7-\mathrm{H} 7$ & $0.947(15)$ & C57-H57 & $0.992(14)$ \\
\hline $\mathrm{C} 7-\mathrm{C} 8$ & $1.3877(12)$ & C57-C58 & $1.3912(13)$ \\
\hline $\mathrm{C} 8-\mathrm{H} 8$ & $1.007(13)$ & C58-H58 & $0.983(14)$ \\
\hline $\mathrm{C} 8-\mathrm{C} 9$ & $1.3988(10)$ & $\mathrm{C} 58-\mathrm{C} 59$ & $1.3982(12)$ \\
\hline $\mathrm{C} 10-\mathrm{H} 10 \mathrm{~A}$ & $1.014(13)$ & $\mathrm{C} 60-\mathrm{H} 60$ & $0.982(13)$ \\
\hline $\mathrm{C} 10-\mathrm{H} 10 \mathrm{~B}$ & $1.001(14)$ & C60-H60B & $0.997(14)$ \\
\hline $\mathrm{C} 10-\mathrm{C} 11$ & $1.5196(12)$ & $\mathrm{C} 60-\mathrm{C} 61$ & $1.5258(14)$ \\
\hline C11-H11A & $0.969(15)$ & C61-H61A & $0.991(16)$ \\
\hline $\mathrm{C} 11-\mathrm{H} 11$ & $0.996(16)$ & C61-H61B & $1.016(14)$ \\
\hline $\mathrm{C} 11-\mathrm{C} 12$ & $1.5275(14)$ & C61-C62 & $1.5269(14)$ \\
\hline $\mathrm{C} 12-\mathrm{H} 12 \mathrm{~A}$ & $0.996(16)$ & C62-H62A & $1.005(18)$ \\
\hline $\mathrm{C} 12-\mathrm{H} 12 \mathrm{~B}$ & $1.023(16)$ & C62-H62B & $0.974(17)$ \\
\hline $\mathrm{C} 12-\mathrm{C} 13$ & $1.5300(15)$ & $\mathrm{C} 62-\mathrm{C} 63$ & $1.5300(16)$ \\
\hline $\mathrm{C} 13-\mathrm{H} 13 \mathrm{~A}$ & $0.998(15)$ & C63-H63A & $0.977(16)$ \\
\hline C13-H13B & $0.979(14)$ & C63-H63B & $0.965(16)$ \\
\hline $\mathrm{C} 13-\mathrm{C} 14$ & $1.5252(12)$ & $\mathrm{C} 63-\mathrm{C} 64$ & $1.5252(14)$ \\
\hline C14-H14A & $0.998(14)$ & C64-H64A & $0.973(14)$ \\
\hline C14-H14B & $0.982(14)$ & C64-H64B & $1.015(14)$ \\
\hline $\mathrm{H} 1 \mathrm{~N}-\mathrm{N} 1-\mathrm{C} 1$ & $114.3(9)$ & $\mathrm{H} 51 \mathrm{~N}-\mathrm{N} 51-\mathrm{C} 51$ & $115.8(10)$ \\
\hline $\mathrm{H} 1 \mathrm{~N}-\mathrm{N} 1-\mathrm{C} 9$ & $115.7(9)$ & $\mathrm{H} 51 \mathrm{~N}-\mathrm{N} 51-\mathrm{C} 59$ & $114.1(10)$ \\
\hline $\mathrm{C} 1-\mathrm{N} 1-\mathrm{C} 9$ & $129.81(6)$ & $\mathrm{C} 51-\mathrm{N} 51-\mathrm{C} 59$ & $130.00(7)$ \\
\hline $\mathrm{N} 3-\mathrm{N} 2-\mathrm{C} 3$ & $105.55(6)$ & $\mathrm{N} 53-\mathrm{N} 52-\mathrm{C} 53$ & $105.52(7)$ \\
\hline $\mathrm{N} 2-\mathrm{N} 3-\mathrm{N} 4$ & $111.36(6)$ & $\mathrm{N} 52-\mathrm{N} 53-\mathrm{N} 54$ & $111.29(7)$ \\
\hline $\mathrm{N} 3-\mathrm{N} 4-\mathrm{N} 5$ & $106.08(6)$ & N53-N54-N55 & $106.29(7)$ \\
\hline $\mathrm{N} 4-\mathrm{N} 5-\mathrm{C} 3$ & $108.10(6)$ & N54-N55-C53 & $107.76(7)$ \\
\hline $\mathrm{N} 4-\mathrm{N} 5-\mathrm{C} 4$ & $122.45(6)$ & N54-N55-C54 & $122.40(7)$ \\
\hline $\mathrm{C} 3-\mathrm{N} 5-\mathrm{C} 4$ & $129.44(6)$ & $\mathrm{C} 53-\mathrm{N} 55-\mathrm{C} 54$ & $129.82(7)$ \\
\hline $\mathrm{C} 2-\mathrm{N} 6-\mathrm{C} 10$ & $111.02(6)$ & $\mathrm{C} 52-\mathrm{N} 56-\mathrm{C} 60$ & $113.57(7)$ \\
\hline $\mathrm{C} 2-\mathrm{N} 6-\mathrm{C} 14$ & $112.06(6)$ & $\mathrm{C} 52-\mathrm{N} 56-\mathrm{C} 64$ & $110.57(6)$ \\
\hline $\mathrm{C} 10-\mathrm{N} 6-\mathrm{C} 14$ & $109.94(6)$ & $\mathrm{C} 60-\mathrm{N} 56-\mathrm{C} 64$ & $109.24(7)$ \\
\hline $\mathrm{O} 1-\mathrm{C} 1-\mathrm{N} 1$ & $121.56(7)$ & $\mathrm{O} 51-\mathrm{C} 51-\mathrm{N} 51$ & $121.40(7)$ \\
\hline $\mathrm{O} 1-\mathrm{C} 1-\mathrm{C} 2$ & $120.43(6)$ & $\mathrm{O} 51-\mathrm{C} 51-\mathrm{C} 52$ & $119.90(7)$ \\
\hline $\mathrm{N} 1-\mathrm{C} 1-\mathrm{C} 2$ & $118.00(6)$ & $\mathrm{N} 51-\mathrm{C} 51-\mathrm{C} 52$ & $118.64(7)$ \\
\hline
\end{tabular}




\begin{tabular}{|c|c|}
\hline $\mathrm{N} 6-\mathrm{C} 2-\mathrm{C} 1$ & $111.27(6)$ \\
\hline $\mathrm{N} 6-\mathrm{C} 2-\mathrm{H} 2$ & $113.6(7)$ \\
\hline $\mathrm{N} 6-\mathrm{C} 2-\mathrm{C} 3$ & $109.40(6)$ \\
\hline $\mathrm{C} 1-\mathrm{C} 2-\mathrm{H} 2$ & $108.1(7)$ \\
\hline $\mathrm{C} 1-\mathrm{C} 2-\mathrm{C} 3$ & $107.10(6)$ \\
\hline $\mathrm{H} 2-\mathrm{C} 2-\mathrm{C} 3$ & $107.0(7)$ \\
\hline $\mathrm{N} 2-\mathrm{C} 3-\mathrm{N} 5$ & $108.91(6)$ \\
\hline $\mathrm{N} 2-\mathrm{C} 3-\mathrm{C} 2$ & $128.45(7)$ \\
\hline $\mathrm{N} 5-\mathrm{C} 3-\mathrm{C} 2$ & $122.63(6)$ \\
\hline $\mathrm{N} 5-\mathrm{C} 4-\mathrm{C} 5$ & $118.89(7)$ \\
\hline $\mathrm{N} 5-\mathrm{C} 4-\mathrm{C} 9$ & $119.78(6)$ \\
\hline $\mathrm{C} 5-\mathrm{C} 4-\mathrm{C} 9$ & $121.33(7)$ \\
\hline $\mathrm{C} 4-\mathrm{C} 5-\mathrm{H} 5$ & $119.5(8)$ \\
\hline $\mathrm{C} 4-\mathrm{C} 5-\mathrm{C} 6$ & $119.48(7)$ \\
\hline $\mathrm{H} 5-\mathrm{C} 5-\mathrm{C} 6$ & $121.0(8)$ \\
\hline $\mathrm{C} 5-\mathrm{C} 6-\mathrm{H} 6$ & $121.3(9)$ \\
\hline $\mathrm{C} 5-\mathrm{C} 6-\mathrm{C} 7$ & $119.99(7)$ \\
\hline $\mathrm{H} 6-\mathrm{C} 6-\mathrm{C} 7$ & $118.7(9)$ \\
\hline $\mathrm{C} 6-\mathrm{C} 7-\mathrm{H} 7$ & $120.5(9)$ \\
\hline $\mathrm{C} 6-\mathrm{C} 7-\mathrm{C} 8$ & $120.13(7)$ \\
\hline $\mathrm{H} 7-\mathrm{C} 7-\mathrm{C} 8$ & $119.4(9)$ \\
\hline $\mathrm{C} 7-\mathrm{C} 8-\mathrm{H} 8$ & $121.2(8)$ \\
\hline $\mathrm{C} 7-\mathrm{C} 8-\mathrm{C} 9$ & $120.79(7)$ \\
\hline $\mathrm{H} 8-\mathrm{C} 8-\mathrm{C} 9$ & $118.0(8)$ \\
\hline $\mathrm{N} 1-\mathrm{C} 9-\mathrm{C} 4$ & $123.90(6)$ \\
\hline $\mathrm{N} 1-\mathrm{C} 9-\mathrm{C} 8$ & $117.64(7)$ \\
\hline $\mathrm{C} 4-\mathrm{C} 9-\mathrm{C} 8$ & $118.27(7)$ \\
\hline $\mathrm{N} 6-\mathrm{C} 10-\mathrm{H} 10 \mathrm{~A}$ & $109.2(7)$ \\
\hline $\mathrm{N} 6-\mathrm{C} 10-\mathrm{H} 10 \mathrm{~B}$ & $109.5(8)$ \\
\hline $\mathrm{N} 6-\mathrm{C} 10-\mathrm{C} 11$ & $109.81(7)$ \\
\hline $\mathrm{H} 10 \mathrm{~A}-\mathrm{C} 10-\mathrm{H} 10 \mathrm{~B}$ & $105.0(11)$ \\
\hline $\mathrm{H} 10 \mathrm{~A}-\mathrm{C} 10-\mathrm{C} 11$ & $111.8(8)$ \\
\hline $\mathrm{H} 10 \mathrm{~B}-\mathrm{C} 10-\mathrm{C} 11$ & $111.4(8)$ \\
\hline $\mathrm{C} 10-\mathrm{C} 11-\mathrm{H} 11 \mathrm{~A}$ & $108.7(9)$ \\
\hline $\mathrm{C} 10-\mathrm{C} 11-\mathrm{H} 11$ & $108.8(9)$ \\
\hline $\mathrm{C} 10-\mathrm{C} 11-\mathrm{C} 12$ & $110.94(8)$ \\
\hline $\mathrm{H} 11 \mathrm{~A}-\mathrm{C} 11-\mathrm{H} 11$ & $107.3(13)$ \\
\hline $\mathrm{H} 11 \mathrm{~A}-\mathrm{C} 11-\mathrm{C} 12$ & $111.0(9)$ \\
\hline $\mathrm{H} 11-\mathrm{C} 11-\mathrm{C} 12$ & $109.9(9)$ \\
\hline $\mathrm{C} 11-\mathrm{C} 12-\mathrm{H} 12 \mathrm{~A}$ & $110.7(9)$ \\
\hline $\mathrm{C} 11-\mathrm{C} 12-\mathrm{H} 12 \mathrm{~B}$ & $110.5(9)$ \\
\hline $\mathrm{C} 11-\mathrm{C} 12-\mathrm{C} 13$ & $110.97(8)$ \\
\hline $\mathrm{H} 12 \mathrm{~A}-\mathrm{C} 12-\mathrm{H} 12 \mathrm{~B}$ & $105.6(13)$ \\
\hline $\mathrm{H} 12 \mathrm{~A}-\mathrm{C} 12-\mathrm{C} 13$ & $108.5(9)$ \\
\hline $\mathrm{H} 12 \mathrm{~B}-\mathrm{C} 12-\mathrm{C} 13$ & $110.4(9)$ \\
\hline $\mathrm{C} 12-\mathrm{C} 13-\mathrm{H} 13 \mathrm{~A}$ & $110.2(9)$ \\
\hline $\mathrm{C} 12-\mathrm{C} 13-\mathrm{H} 13 \mathrm{~B}$ & $110.7(8)$ \\
\hline $\mathrm{C} 12-\mathrm{C} 13-\mathrm{C} 14$ & $111.35(8)$ \\
\hline
\end{tabular}

\begin{tabular}{|c|c|}
\hline $\mathrm{N} 56-\mathrm{C} 52-\mathrm{C} 51$ & $113.38(7)$ \\
\hline N56-C52-H52 & $113.6(7)$ \\
\hline $\mathrm{N} 56-\mathrm{C} 52-\mathrm{C} 53$ & $108.84(7)$ \\
\hline $\mathrm{C} 51-\mathrm{C} 52-\mathrm{H} 52$ & $105.9(7)$ \\
\hline $\mathrm{C} 51-\mathrm{C} 52-\mathrm{C} 53$ & $105.63(6)$ \\
\hline $\mathrm{H} 52-\mathrm{C} 52-\mathrm{C} 53$ & $109.1(7)$ \\
\hline $\mathrm{N} 52-\mathrm{C} 53-\mathrm{N} 55$ & $109.13(7)$ \\
\hline $\mathrm{N} 52-\mathrm{C} 53-\mathrm{C} 52$ & $128.94(8)$ \\
\hline $\mathrm{N} 55-\mathrm{C} 53-\mathrm{C} 52$ & $121.92(7)$ \\
\hline $\mathrm{N} 55-\mathrm{C} 54-\mathrm{C} 55$ & $119.38(7)$ \\
\hline $\mathrm{N} 55-\mathrm{C} 54-\mathrm{C} 59$ & $119.52(7)$ \\
\hline $\mathrm{C} 55-\mathrm{C} 54-\mathrm{C} 59$ & $121.06(8)$ \\
\hline $\mathrm{C} 54-\mathrm{C} 55-\mathrm{H} 55$ & $120.3(8)$ \\
\hline $\mathrm{C} 54-\mathrm{C} 55-\mathrm{C} 56$ & $119.71(8)$ \\
\hline $\mathrm{H} 55-\mathrm{C} 55-\mathrm{C} 56$ & $119.9(8)$ \\
\hline $\mathrm{C} 55-\mathrm{C} 56-\mathrm{H} 56$ & $120.7(9)$ \\
\hline $\mathrm{C} 55-\mathrm{C} 56-\mathrm{C} 57$ & $119.95(8)$ \\
\hline $\mathrm{H} 56-\mathrm{C} 56-\mathrm{C} 57$ & $119.3(9)$ \\
\hline $\mathrm{C} 56-\mathrm{C} 57-\mathrm{H} 57$ & $122.7(8)$ \\
\hline $\mathrm{C} 56-\mathrm{C} 57-\mathrm{C} 58$ & $120.12(8)$ \\
\hline $\mathrm{H} 57-\mathrm{C} 57-\mathrm{C} 58$ & $117.2(8)$ \\
\hline $\mathrm{C} 57-\mathrm{C} 58-\mathrm{H} 58$ & $119.8(8)$ \\
\hline $\mathrm{C} 57-\mathrm{C} 58-\mathrm{C} 59$ & $120.60(8)$ \\
\hline $\mathrm{H} 58-\mathrm{C} 58-\mathrm{C} 59$ & $119.6(8)$ \\
\hline $\mathrm{N} 51-\mathrm{C} 59-\mathrm{C} 54$ & $123.27(7)$ \\
\hline $\mathrm{N} 51-\mathrm{C} 59-\mathrm{C} 58$ & $117.95(7)$ \\
\hline $\mathrm{C} 54-\mathrm{C} 59-\mathrm{C} 58$ & $118.55(7)$ \\
\hline $\mathrm{N} 56-\mathrm{C} 60-\mathrm{H} 60$ & $109.4(8)$ \\
\hline $\mathrm{N} 56-\mathrm{C} 60-\mathrm{H} 60 \mathrm{~B}$ & $108.1(8)$ \\
\hline $\mathrm{N} 56-\mathrm{C} 60-\mathrm{C} 61$ & $108.76(7)$ \\
\hline $\mathrm{H} 60-\mathrm{C} 60-\mathrm{H} 60 \mathrm{~B}$ & $111.6(11)$ \\
\hline $\mathrm{H} 60-\mathrm{C} 60-\mathrm{C} 61$ & $109.8(8)$ \\
\hline $\mathrm{H} 60 \mathrm{~B}-\mathrm{C} 60-\mathrm{C} 61$ & $109.2(8)$ \\
\hline $\mathrm{C} 60-\mathrm{C} 61-\mathrm{H} 61 \mathrm{~A}$ & $108.9(9)$ \\
\hline $\mathrm{C} 60-\mathrm{C} 61-\mathrm{H} 61 \mathrm{~B}$ & $109.5(8)$ \\
\hline $\mathrm{C} 60-\mathrm{C} 61-\mathrm{C} 62$ & $111.39(8)$ \\
\hline $\mathrm{H} 61 \mathrm{~A}-\mathrm{C} 61-\mathrm{H} 61 \mathrm{~B}$ & $107.4(12)$ \\
\hline H61A-C61-C62 & $110.2(9)$ \\
\hline $\mathrm{H} 61 \mathrm{~B}-\mathrm{C} 61-\mathrm{C} 62$ & $109.4(8)$ \\
\hline $\mathrm{C} 61-\mathrm{C} 62-\mathrm{H} 62 \mathrm{~A}$ & $109.2(10)$ \\
\hline C61-C62-H62B & $109.1(9)$ \\
\hline $\mathrm{C} 61-\mathrm{C} 62-\mathrm{C} 63$ & $110.61(8)$ \\
\hline $\mathrm{H} 62 \mathrm{~A}-\mathrm{C} 62-\mathrm{H} 62 \mathrm{~B}$ & $102.9(13)$ \\
\hline $\mathrm{H} 62 \mathrm{~A}-\mathrm{C} 62-\mathrm{C} 63$ & $110.7(10)$ \\
\hline $\mathrm{H} 62 \mathrm{~B}-\mathrm{C} 62-\mathrm{C} 63$ & $114.0(9)$ \\
\hline $\mathrm{C} 62-\mathrm{C} 63-\mathrm{H} 63 \mathrm{~A}$ & $111.7(10)$ \\
\hline $\mathrm{C} 62-\mathrm{C} 63-\mathrm{H} 63 \mathrm{~B}$ & $111.2(9)$ \\
\hline C62-C63-C64 & $110.88(9)$ \\
\hline
\end{tabular}




\begin{tabular}{|c|c|c|c|}
\hline $\mathrm{H} 13 \mathrm{~A}-\mathrm{C} 13-\mathrm{H} 13 \mathrm{~B}$ & $110.0(12)$ & $\mathrm{H} 63 \mathrm{~A}-\mathrm{C} 63-\mathrm{H} 63 \mathrm{~B}$ & $105.8(13)$ \\
\hline $\mathrm{H} 13 \mathrm{~A}-\mathrm{C} 13-\mathrm{C} 14$ & $106.0(8)$ & $\mathrm{H} 63 \mathrm{~A}-\mathrm{C} 63-\mathrm{C} 64$ & $107.8(9)$ \\
\hline $\mathrm{H} 13 \mathrm{~B}-\mathrm{C} 13-\mathrm{C} 14$ & $108.4(8)$ & H63B-C63-C64 & $109.4(9)$ \\
\hline $\mathrm{N} 6-\mathrm{C} 14-\mathrm{C} 13$ & $109.53(7)$ & $\mathrm{N} 56-\mathrm{C} 64-\mathrm{C} 63$ & $110.32(7)$ \\
\hline $\mathrm{N} 6-\mathrm{C} 14-\mathrm{H} 14 \mathrm{~A}$ & $107.6(8)$ & N56-C64-H64A & $108.6(8)$ \\
\hline $\mathrm{N} 6-\mathrm{C} 14-\mathrm{H} 14 \mathrm{~B}$ & $109.8(8)$ & N56-C64-H64B & $108.5(8)$ \\
\hline $\mathrm{C} 13-\mathrm{C} 14-\mathrm{H} 14 \mathrm{~A}$ & $109.5(8)$ & C63-C64-H64A & $110.7(8)$ \\
\hline C13-C14-H14B & $109.4(8)$ & C63-C64-H64B & $111.4(8)$ \\
\hline $\mathrm{H} 14 \mathrm{~A}-\mathrm{C} 14-\mathrm{H} 14 \mathrm{~B}$ & $111.0(12)$ & $\mathrm{H} 64 \mathrm{~A}-\mathrm{C} 64-\mathrm{H} 64 \mathrm{~B}$ & $107.2(12)$ \\
\hline $\mathrm{C} 3-\mathrm{N} 2-\mathrm{N} 3-\mathrm{N} 4$ & $0.15(9)$ & $\mathrm{C} 53-\mathrm{N} 52-\mathrm{N} 53-\mathrm{N} 54$ & $0.19(10)$ \\
\hline $\mathrm{N} 2-\mathrm{N} 3-\mathrm{N} 4-\mathrm{N} 5$ & $0.29(8)$ & N52-N53-N54-N55 & $0.05(10)$ \\
\hline $\mathrm{N} 3-\mathrm{N} 4-\mathrm{N} 5-\mathrm{C} 3$ & $-0.62(8)$ & $\mathrm{N} 53-\mathrm{N} 54-\mathrm{N} 55-\mathrm{C} 53$ & $-0.27(9)$ \\
\hline $\mathrm{N} 3-\mathrm{N} 4-\mathrm{N} 5-\mathrm{C} 4$ & $-179.48(7)$ & N53-N54-N55-C54 & $177.97(8)$ \\
\hline $\mathrm{C} 9-\mathrm{N} 1-\mathrm{C} 1-\mathrm{O} 1$ & $179.78(7)$ & $\mathrm{C} 59-\mathrm{N} 51-\mathrm{C} 51-\mathrm{O} 51$ & $178.22(9)$ \\
\hline $\mathrm{C} 9-\mathrm{N} 1-\mathrm{C} 1-\mathrm{C} 2$ & $1.11(11)$ & $\mathrm{C} 59-\mathrm{N} 51-\mathrm{C} 51-\mathrm{C} 52$ & $0.99(14)$ \\
\hline $\mathrm{C} 10-\mathrm{N} 6-\mathrm{C} 2-\mathrm{C} 1$ & $-178.53(6)$ & $\mathrm{C} 60-\mathrm{N} 56-\mathrm{C} 52-\mathrm{C} 51$ & $-50.44(9)$ \\
\hline $\mathrm{C} 10-\mathrm{N} 6-\mathrm{C} 2-\mathrm{C} 3$ & $63.32(7)$ & $\mathrm{C} 60-\mathrm{N} 56-\mathrm{C} 52-\mathrm{C} 53$ & $-167.67(6)$ \\
\hline $\mathrm{C} 14-\mathrm{N} 6-\mathrm{C} 2-\mathrm{C} 1$ & $-55.17(8)$ & $\mathrm{C} 64-\mathrm{N} 56-\mathrm{C} 52-\mathrm{C} 51$ & $-173.65(7)$ \\
\hline $\mathrm{C} 14-\mathrm{N} 6-\mathrm{C} 2-\mathrm{C} 3$ & $-173.31(6)$ & $\mathrm{C} 64-\mathrm{N} 56-\mathrm{C} 52-\mathrm{C} 53$ & $69.12(8)$ \\
\hline $\mathrm{O} 1-\mathrm{C} 1-\mathrm{C} 2-\mathrm{N} 6$ & $123.80(7)$ & $\mathrm{O} 51-\mathrm{C} 51-\mathrm{C} 52-\mathrm{N} 56$ & $126.51(9)$ \\
\hline $\mathrm{O} 1-\mathrm{C} 1-\mathrm{C} 2-\mathrm{C} 3$ & $-116.68(7)$ & $\mathrm{O} 51-\mathrm{C} 51-\mathrm{C} 52-\mathrm{C} 53$ & $-114.40(9)$ \\
\hline $\mathrm{N} 1-\mathrm{C} 1-\mathrm{C} 2-\mathrm{N} 6$ & $-57.52(8)$ & $\mathrm{N} 51-\mathrm{C} 51-\mathrm{C} 52-\mathrm{N} 56$ & $-56.22(10)$ \\
\hline $\mathrm{N} 1-\mathrm{C} 1-\mathrm{C} 2-\mathrm{C} 3$ & $62.01(8)$ & $\mathrm{N} 51-\mathrm{C} 51-\mathrm{C} 52-\mathrm{C} 53$ & $62.87(10)$ \\
\hline $\mathrm{N} 3-\mathrm{N} 2-\mathrm{C} 3-\mathrm{N} 5$ & $-0.53(8)$ & $\mathrm{N} 53-\mathrm{N} 52-\mathrm{C} 53-\mathrm{N} 55$ & $-0.36(9)$ \\
\hline $\mathrm{N} 3-\mathrm{N} 2-\mathrm{C} 3-\mathrm{C} 2$ & $178.55(7)$ & $\mathrm{N} 53-\mathrm{N} 52-\mathrm{C} 53-\mathrm{C} 52$ & $-179.19(8)$ \\
\hline $\mathrm{N} 4-\mathrm{N} 5-\mathrm{C} 3-\mathrm{N} 2$ & $0.73(8)$ & N54-N55-C53-N52 & $0.40(9)$ \\
\hline $\mathrm{N} 4-\mathrm{N} 5-\mathrm{C} 3-\mathrm{C} 2$ & $-178.43(6)$ & $\mathrm{N} 54-\mathrm{N} 55-\mathrm{C} 53-\mathrm{C} 52$ & $179.32(7)$ \\
\hline $\mathrm{C} 4-\mathrm{N} 5-\mathrm{C} 3-\mathrm{N} 2$ & $179.49(7)$ & $\mathrm{C} 54-\mathrm{N} 55-\mathrm{C} 53-\mathrm{N} 52$ & $-177.66(8)$ \\
\hline $\mathrm{C} 4-\mathrm{N} 5-\mathrm{C} 3-\mathrm{C} 2$ & $0.33(12)$ & $\mathrm{C} 54-\mathrm{N} 55-\mathrm{C} 53-\mathrm{C} 52$ & $1.26(13)$ \\
\hline $\mathrm{N} 6-\mathrm{C} 2-\mathrm{C} 3-\mathrm{N} 2$ & $-121.66(8)$ & N56-C52-C53-N52 & $-124.11(9)$ \\
\hline $\mathrm{N} 6-\mathrm{C} 2-\mathrm{C} 3-\mathrm{N} 5$ & $57.31(9)$ & $\mathrm{N} 56-\mathrm{C} 52-\mathrm{C} 53-\mathrm{N} 55$ & $57.19(10)$ \\
\hline $\mathrm{C} 1-\mathrm{C} 2-\mathrm{C} 3-\mathrm{N} 2$ & $117.62(8)$ & $\mathrm{C} 51-\mathrm{C} 52-\mathrm{C} 53-\mathrm{N} 52$ & $113.82(9)$ \\
\hline $\mathrm{C} 1-\mathrm{C} 2-\mathrm{C} 3-\mathrm{N} 5$ & $-63.41(9)$ & $\mathrm{C} 51-\mathrm{C} 52-\mathrm{C} 53-\mathrm{N} 55$ & $-64.87(10)$ \\
\hline $\mathrm{N} 4-\mathrm{N} 5-\mathrm{C} 4-\mathrm{C} 5$ & $33.23(10)$ & N54-N55-C54-C55 & $36.85(11)$ \\
\hline $\mathrm{N} 4-\mathrm{N} 5-\mathrm{C} 4-\mathrm{C} 9$ & $-146.46(7)$ & N54-N55-C54-C59 & $-140.99(8)$ \\
\hline $\mathrm{C} 3-\mathrm{N} 5-\mathrm{C} 4-\mathrm{C} 5$ & $-145.37(8)$ & $\mathrm{C} 53-\mathrm{N} 55-\mathrm{C} 54-\mathrm{C} 55$ & $-145.34(9)$ \\
\hline $\mathrm{C} 3-\mathrm{N} 5-\mathrm{C} 4-\mathrm{C} 9$ & $34.94(11)$ & C53-N55-C54-C59 & $36.82(12)$ \\
\hline $\mathrm{N} 5-\mathrm{C} 4-\mathrm{C} 5-\mathrm{C} 6$ & $179.98(7)$ & N55-C54-C55-C56 & $-177.01(8)$ \\
\hline $\mathrm{C} 9-\mathrm{C} 4-\mathrm{C} 5-\mathrm{C} 6$ & $-0.34(12)$ & $\mathrm{C} 59-\mathrm{C} 54-\mathrm{C} 55-\mathrm{C} 56$ & $0.80(13)$ \\
\hline $\mathrm{C} 4-\mathrm{C} 5-\mathrm{C} 6-\mathrm{C} 7$ & $-0.64(12)$ & $\mathrm{C} 54-\mathrm{C} 55-\mathrm{C} 56-\mathrm{C} 57$ & $-0.99(13)$ \\
\hline $\mathrm{C} 5-\mathrm{C} 6-\mathrm{C} 7-\mathrm{C} 8$ & $0.98(13)$ & $\mathrm{C} 55-\mathrm{C} 56-\mathrm{C} 57-\mathrm{C} 58$ & $0.65(14)$ \\
\hline $\mathrm{C} 6-\mathrm{C} 7-\mathrm{C} 8-\mathrm{C} 9$ & $-0.34(13)$ & $\mathrm{C} 56-\mathrm{C} 57-\mathrm{C} 58-\mathrm{C} 59$ & $-0.11(14)$ \\
\hline $\mathrm{C} 7-\mathrm{C} 8-\mathrm{C} 9-\mathrm{N} 1$ & $174.59(7)$ & $\mathrm{C} 57-\mathrm{C} 58-\mathrm{C} 59-\mathrm{N} 51$ & $174.60(8)$ \\
\hline $\mathrm{C} 7-\mathrm{C} 8-\mathrm{C} 9-\mathrm{C} 4$ & $-0.62(11)$ & $\mathrm{C} 57-\mathrm{C} 58-\mathrm{C} 59-\mathrm{C} 54$ & $-0.09(13)$ \\
\hline $\mathrm{N} 5-\mathrm{C} 4-\mathrm{C} 9-\mathrm{N} 1$ & $5.76(11)$ & N55-C54-C59-N51 & $3.16(12)$ \\
\hline $\mathrm{N} 5-\mathrm{C} 4-\mathrm{C} 9-\mathrm{C} 8$ & $-179.36(7)$ & N55-C54-C59-C58 & $177.55(7)$ \\
\hline $\mathrm{C} 5-\mathrm{C} 4-\mathrm{C} 9-\mathrm{N} 1$ & $-173.92(7)$ & $\mathrm{C} 55-\mathrm{C} 54-\mathrm{C} 59-\mathrm{N} 51$ & $-174.65(8)$ \\
\hline
\end{tabular}




$\begin{array}{llll}\mathrm{C} 5-\mathrm{C} 4-\mathrm{C} 9-\mathrm{C} 8 & 0.96(11) & \mathrm{C} 55-\mathrm{C} 54-\mathrm{C} 59-\mathrm{C} 58 & -0.26(12) \\ \mathrm{C} 1-\mathrm{N} 1-\mathrm{C} 9-\mathrm{C} 4 & -43.24(12) & \mathrm{C} 51-\mathrm{N} 51-\mathrm{C} 59-\mathrm{C} 54 & -41.97(13) \\ \mathrm{C} 1-\mathrm{N} 1-\mathrm{C} 9-\mathrm{C} 8 & 141.84(8) & \mathrm{C} 51-\mathrm{N} 51-\mathrm{C} 59-\mathrm{C} 58 & 143.61(9) \\ \mathrm{C} 2-\mathrm{N} 6-\mathrm{C} 10-\mathrm{C} 11 & -171.45(6) & \mathrm{C} 52-\mathrm{N} 56-\mathrm{C} 60-\mathrm{C} 61 & 171.56(7) \\ \mathrm{C} 14-\mathrm{N} 6-\mathrm{C} 10-\mathrm{C} 11 & 63.98(8) & \mathrm{C} 64-\mathrm{N} 56-\mathrm{C} 60-\mathrm{C} 61 & -64.50(9) \\ \mathrm{N} 6-\mathrm{C} 10-\mathrm{C} 11-\mathrm{C} 12 & -57.34(10) & \mathrm{N} 56-\mathrm{C} 60-\mathrm{C} 61-\mathrm{C} 62 & 58.95(10) \\ \mathrm{C} 10-\mathrm{C} 11-\mathrm{C} 12-\mathrm{C} 13 & 50.96(11) & \mathrm{C} 60-\mathrm{C} 61-\mathrm{C} 62-\mathrm{C} 63 & -52.02(12) \\ \mathrm{C} 11-\mathrm{C} 12-\mathrm{C} 13-\mathrm{C} 14 & -50.81(11) & \mathrm{C} 61-\mathrm{C} 62-\mathrm{C} 63-\mathrm{C} 64 & 50.42(12) \\ \mathrm{C} 2-\mathrm{N} 6-\mathrm{C} 14-\mathrm{C} 13 & 172.62(7) & \mathrm{C} 52-\mathrm{N} 56-\mathrm{C} 64-\mathrm{C} 63 & -170.26(8) \\ \mathrm{C} 10-\mathrm{N} 6-\mathrm{C} 14-\mathrm{C} 13 & -63.41(9) & \mathrm{C} 60-\mathrm{N} 56-\mathrm{C} 64-\mathrm{C} 63 & 64.06(10) \\ \mathrm{C} 12-\mathrm{C} 13-\mathrm{C} 14-\mathrm{N} 6 & 56.84(10) & \mathrm{C} 62-\mathrm{C} 63-\mathrm{C} 64-\mathrm{N} 56 & -56.70(11)\end{array}$

Hydrogen-bond geometry $\left(A,{ }^{\circ}\right)$

\begin{tabular}{lllll}
\hline$D-\mathrm{H} \cdots A$ & $D-\mathrm{H}$ & $\mathrm{H} \cdots A$ & $D \cdots A$ & $D-\mathrm{H} \cdots A$ \\
\hline $\mathrm{N} 1-\mathrm{H} 1 N \cdots \mathrm{O} 1^{\mathrm{i}}$ & $0.850(14)$ & $2.069(14)$ & $2.9089(9)$ & $169.4(13)$ \\
$\mathrm{N} 51-\mathrm{H} 51 N \cdots \mathrm{O} 51^{\mathrm{ii}}$ & $0.886(16)$ & $1.929(16)$ & $2.8116(10)$ & $173.6(14)$ \\
$\mathrm{C} 6-\mathrm{H} 6 \cdots 2^{\mathrm{iii}}$ & $0.936(15)$ & $2.531(15)$ & $3.4638(11)$ & $174.8(12)$ \\
$\mathrm{C} 7-\mathrm{H} 7 \cdots \mathrm{O} 1^{\mathrm{iii}}$ & $0.947(15)$ & $2.406(15)$ & $3.3394(10)$ & $168.4(13)$ \\
$\mathrm{C} 55-\mathrm{H} 55 \cdots \mathrm{N} 54^{\mathrm{iv}}$ & $0.974(13)$ & $2.548(13)$ & $3.2293(11)$ & $127.0(10)$
\end{tabular}

Symmetry codes: (i) $-x+1,-y,-z+1$; (ii) $-x+1,-y+1,-z$; (iii) $x+1, y, z$; (iv) $-x+1,-y+1,-z+1$. 